Z. klin. Chem. u. klin. Biochem.

7. Jg., S. 42-44, Januar 1969

\title{
Zur Frage eines Inhibitors der Benzoylcholinhydrolase (EC 3.1.1.9) im Blutplasma des Menschen')
}

\author{
Von Hildegard Weiss und M. Meyer \\ Aus dem Institut für Krebsforschung der Deutschen Akademie der Wissenschaften ₹u Berlin, \\ Bereich Robert-Rössle-Klinik (Direktor: Prof. Dr. H. Gum mel)
}

(Eingegangen am 21. September 1968)

Für die Aktivierung der Benzoylcholinhydrolase des Gesamtplasmas durch Hydroxylamin wurde eine Konzentrationsabhängigkeit gefunden.

Der aktivitätssteigernde Alterungseffekt konnte den durch Gelfiltration isolierten Isoenzymfraktionen $x, C_{3}$ und $C_{2}$ zugeordnet werden und erwies sich für dieše Fraktionen als $\mathrm{Mg}^{++}$und EDTA unabhängig.

Auf die Aktivierung dutch Hydroxylamin sprechen ebenfalls die Isoenzymfraktionen $x, C_{3}$ und $C_{2}$ besonders an.

\section{Investigations of an inbibitor of benzoyl cboline bydrolase (EC 3.1.1.9) in buman blood plasma}

The activation of benzoyl choline hydrolase of whole plasma by hydroxylamine was found to be concentration-dependent. The increase of activity with age is attributed to the isoenzyme fractions $x, C_{3}$ and $C_{2}$, which were isolated by gelfiltration, and for these fractions the activity was independent of $\mathrm{Mg}^{++}$and EDTA. The activation by hydroxylamine is also exerted especially on isoenzyme fractions $\mathrm{x}, \mathrm{C}_{3}$ and $C_{2}$.

Wie wir (1) 1966 mitteilten, effährt die enzymatische Spaltung von Benzoylcholin durch die Plasmacholinesterase des Menschen eine Erhöhung, wenn das Plasma einige Zeit bei $25^{\circ}$ aufbewahrt wird. FrIEss und Mitarbeiter (2) beobachteten an der Acetylcholinesterase aus dem elektrischen Organ von Electrophorus electricus ebenfalls eine Aktivitätssteigerung durch Alternlassen in Verdünnung. Die Steigerung war aber durch $\mathrm{Mg}^{++}$bzw. EDTA beeinflußbar.

1967 (3) gelang es uns, die $\mathrm{BChH}^{2}$ ) durch Hydroxylamin, das seinerseits ein bekannter Reaktivator für carbamyliertes Enzym ist (4), zu aktivieren.

Im folgenden soll über die Fortführung unserer Untersuchungen berichtet werden: Zunächst wurde die Konzentrationsabhängigkeit der Hydroxylamin-Wirkung bestimmt und im weiteren das Verhalten der durch Sephadex-Gelfiltration getrennten Isoenzyme bezüglich Alterung und deren Beeinflußbarkeit durch $\mathrm{Mg}^{++}$bzw. EDTA geprüft sowie ihre Aktivierbarkeit durch Hÿdroxylamin getestet.

\section{Methodik}

Als Enzymquelle diente Heparinplasma, das aus Spenderblut vor der Abnahme von $400 \mathrm{~m} l$ Blut gewonnen wurde.

Die hydrolytische Spaltung von Benzoylcholin wurde spektrophotometrisch nach KALOw (5) an einem registrierenden UnicamSpektrophotometer SP 800 gemessen. Die Trennung der Isoenzyme erfolgte durch Gelfiltration an Sephadex G 200 in Anlehnung an das von Harris und Robson (6) beschriebene Verfahren.

Die benutzte Hydroxylamin-Lösung wurde aus Hydroxylaminsulfat durch Neutralisation mit $\mathrm{Ba}(\mathrm{OH})_{2}$ hergestellt. Die Kontaktzeit zwischen Ferment und Hydroxylamin vor der Substratzu-

1) Teilweise vorgetragen auf der IV. Jahrestagung der Biochemischen Gesellschaft der DDR (22.-24. 9. 1967) in Rostock.

2) Abkirzungen: $\mathrm{BChH}=$ Benzoylcholinhydrolase (EC 3.1.1.9.) gabe betrug $3 \mathrm{Min}$. In den Meßansätzen der Isoenzyme lag die Konzentration für Hydroxylamin bei $25 \mathrm{~mm}$. Die Endkonzentrationen für $\mathrm{MgCl}_{2}$ und EDTA betrugen $1 \mathrm{~mm}$.

\section{Ergebnisse}

Abbildung 1 zeigt die Zunahme der Benzoylcholinspaltung durch Nativplașma in Abhängigkeit von der zugesetzten Hydroxylamin-Konzentration. Bei einer Konzentration von 7,5 mM ist praktisch eine Sättigung des Ferments mit Hydroxylamin erreicht. Eine Erhöhung der Kontaktzeit zwischen Fermentlösung und Hydroxylamin führte nicht zu gesteigerter Aktivierung. Die Auftrennung der $\mathrm{BChH}$ durch Gelfiltration an Sephadex G 200 ergab 5 Isoenzymfraktionen (s. Abb. 2). Davon entsprechen 4 den von der Stärkegelelektrophorese her bekannten Fraktionen $C_{1}-C_{4}$ $(6,7)$. Ob die 5. Fraktion - von uns mit $x$ bezeichnet mit einer der 7 von LAMotra (8) durch Stärkegelelektrophorese getrennten Fraktionen identisch ist, ist nicht zu sagen.

In Abbildung 3 sind die typischen Aktivitätsveränderungen der einzelnen Enzymfraktionen, die sich durch die Alterung ergeben, aufgetragen. Die Eluate wurden $165 \mathrm{Min}$. bei $25^{\circ}$ gehalten und zur Zeit $t_{0}$ und $t_{165}$ gemessen. Die BChH des Gesamtplasmas erfährt in dieser Zeit nur eine Aktivitätssteigerung von $9 \%$. Dagegen ist der in einer Aktivitätssteigerung entstehende Alterungseffekt bei verschiedenen Isoenzymfraktionen sehr viel ausgeprägter. Er ist typisch für die Fraktionen $x, C_{3}$ und $C_{2}$.

In dem hier aufgezeigten Fall beträgt er $43 \%, 18 \%$ bzw. $93 \%$. Die Fraktionen $C_{4}$ und $C_{1}$ verlieren dagegen an Aktivität. Etwas beeinflußbar ist das Ausmaß der Aktivierung durch den Grad der Verdünnung der Eluate; eine höhere Verdünnung bewirkt eine höhere Aktivierung. 


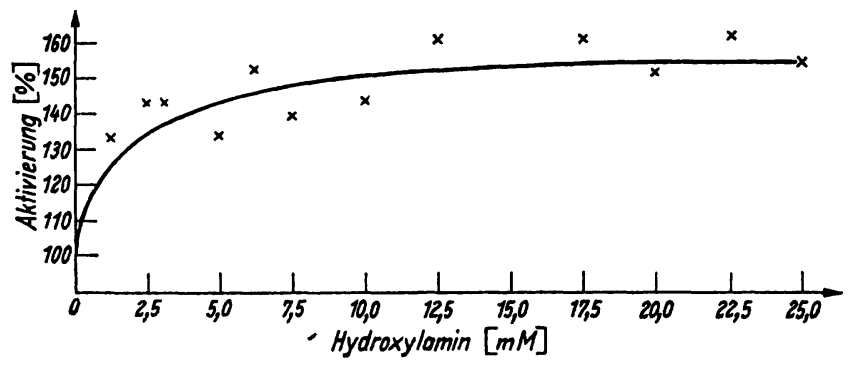

Abb. 1

Abhängigkeit der Aktivierung der ${ }^{\circ} \mathrm{BChH}$ von der HydroxylaminKonzentration

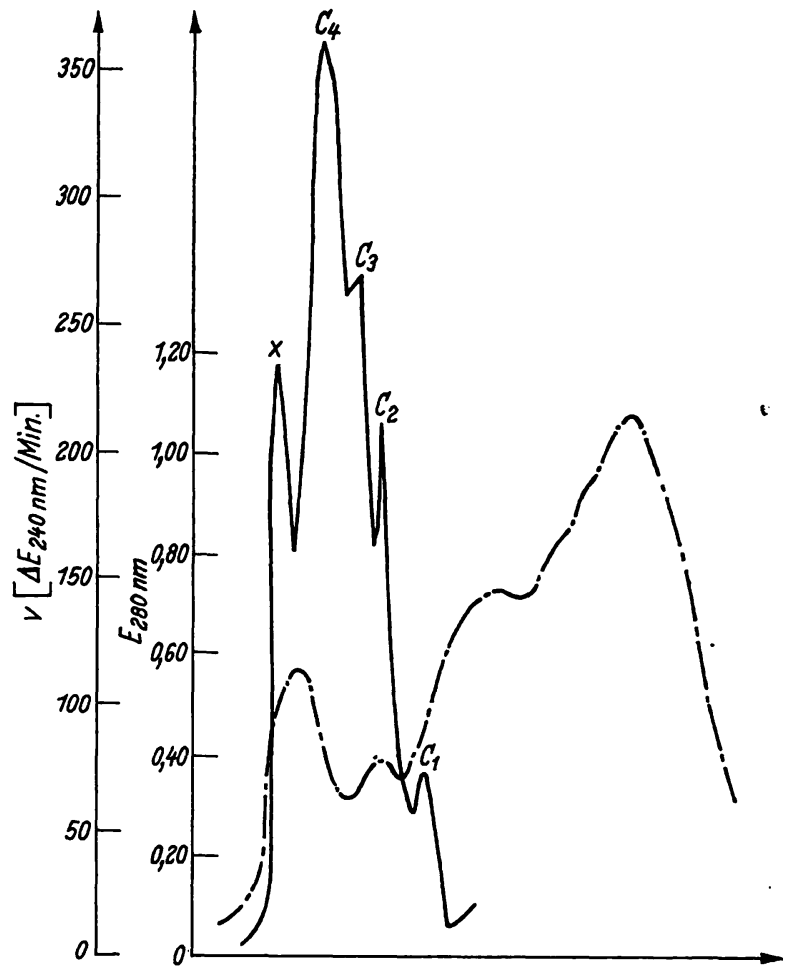

Abb. 2

Ergebnis der Trennung von $2 \mathrm{ml}$ Plasma auf einer Sephadex G 200Säule (50 cm lang, 2,5 cm Durchmesser). Elutionsmittel: $M / 15$ P.hos-

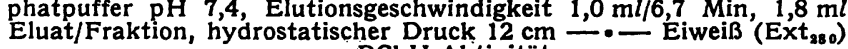
(Das Eluat wurde entsprechend seiner Aktivität mit Phosphatpuffer verdünnt, bei hoher Aktivität bis $1: 50$ )

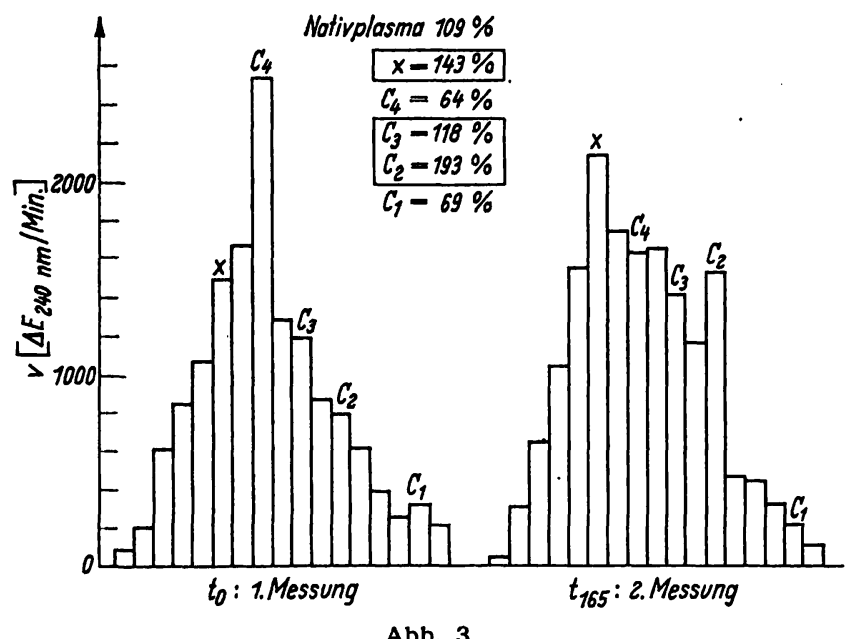

Aktivitätsveränderungen der Isoenzymfraktionen nach Alterung $\left(165\right.$ Min. bei $\left.25^{\circ}\right)$. Meßwerte von $t_{n}=100 \%$

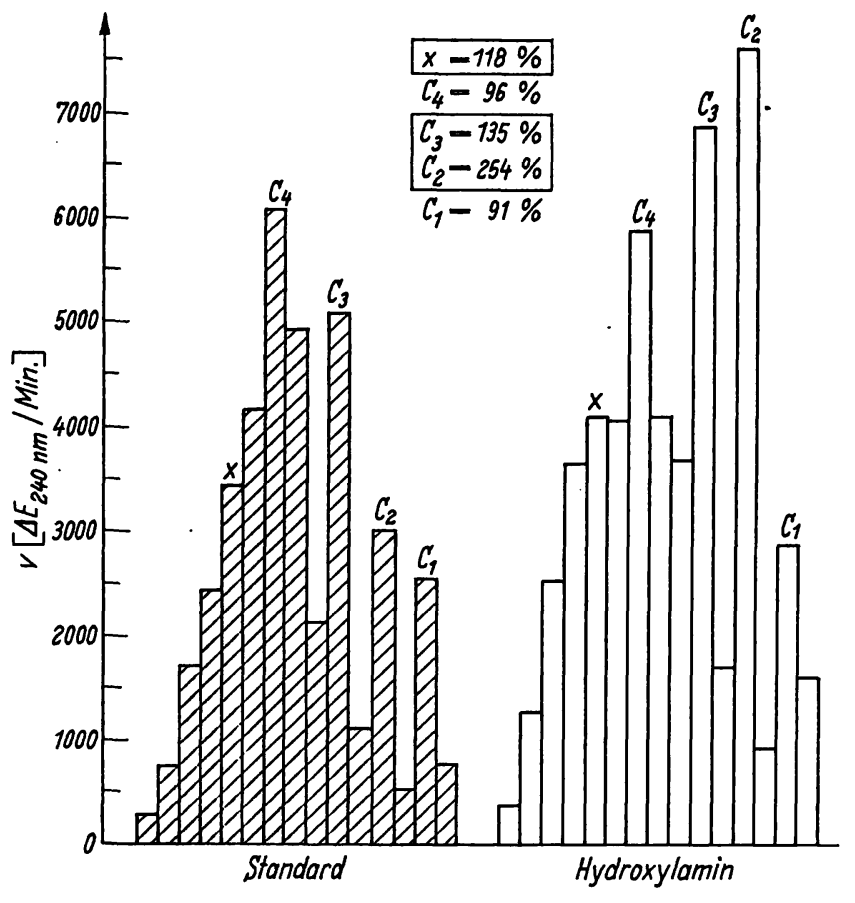

Abb. 5

Verhalten der Isoenzymfraktionen in Anwesenheit von Hydroxylamin $(25 \mathrm{~mm})$. Standardmessungen $=100 \%$

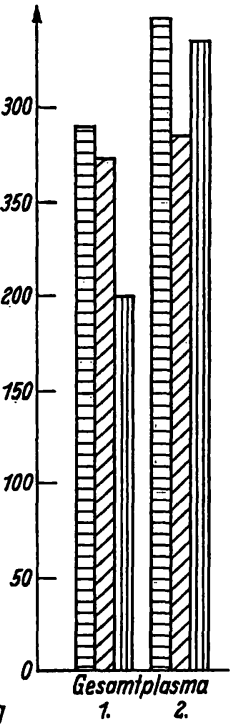

Abb. 4

Beeinflußbarkeit der Aktivitätssteigerung durch Alterung mit $\mathrm{Mg}++$ und EDTA $(0,1 \mathrm{~mm})$

Standard
W/I// $\mathrm{Mg}^{++}$
|||||| EDTA 
Die Wirkung von $\mathrm{Mg}^{++}$und EDTA auf das Gesamtplasma und die einzelnen Isoenzymfraktionen ist in Abbildung 4 dargestellt. EDTA verstärkt den Alterungseffekt bei Gesamtplasma und der $\mathrm{C}_{4}$-Fraktion. $\mathrm{Mg}^{++}$aktiviert nur die $\mathrm{C}_{4}-$ Fraktion in geringem Ausmaß.

Die einzelnen Isoenzymfraktionen wurden auf ihre Aktivierbarkeit durch Hydroxylamin geprüft (s. Abb. 5). Die Fraktionen $x, C_{3}$ und $C_{2}$ zeichnen sich wieder durch besondere Aktivierbarkeit aus; in dem hier gezeigten Fall beträgt sie $18 \%, 35 \%$ bzw. $154 \%$.

\section{Diskussion}

Das Ergebnis der Trennung der $\mathrm{BChH}$ in Isoenzyme läßt sich in die von LAMotra (8) erhobenen und zitierten Befunde einordnen. Ob es sich bei der Aktivitätsänderung durch Altern bei den einzelnen Isoenzymen um eine der von ihm besprochenen Umwandlung der verschiedenen Komponenten $\operatorname{der} \mathrm{BChH}$ handelt, ist nicht zu sagen.
Klar unterschieden werden konnten die Befunde gegen die FrIess'schen Beobachtungen (2), indem die Verstärkung der Alterung durch EDTA bei Gesamtplasma und der $\mathrm{C}_{4}$-Fraktion bestätigt werden konnte, EDTA sich dagegen auf die Fraktionen $x, C_{3}$ und $C_{2}$ - die die für unseren Befund charakteristischen Fraktionen darstellen - als wirkungslos erweist. Die von FriEss gefundene Aktivierung durch $\mathrm{Mg}^{++}$auf einen konstanten Wert, konnte von uns nicht nachgewiesen werden.

Entsprechend der von uns schon früher vertretenen Auffassung (3) scheint es sich sowohl bei der Aktivitätserhöhung durch Altern als auch bei der Aktivierung durch Hydroxylamin um den Zerfall eines FermentInhibitor-Komplexes zu handeln. Mit aller Vorsicht denken wir dabei an eine $\mathrm{CO}_{2}$-Kontrolle des Ferments in vivo; unter Umständen wird über die Bildung von Carbamaten die $\mathrm{BChH}$ gehemmt.

Aktivitätsmessungen der $\mathrm{BChH}$ unter verschiedenem $\mathrm{pCO}_{2}$ laufen zur Zeit in unserem Laboratorium.

\section{Literatur .}

1. MEYER, M. und H. WEIss, Naturwissenschaften 53, 279 (1966). 2. Friess, S. L., J. B. Wilson und E. Caleib, J. Amer. chem. Soc. 76, 5156. (1954). - 3. Werss, H. und M. MEYer, Naturwissenschaften 54, 493 (1967). - 4. WILSON, I. B., M. A. HARRIson und S. Ginsburg, J. biol. Chemistry 236, 1498 (1961). 5. Kalow, W. und H. A. Lindsay, Can. J. Biochem. Physiol. 33, 568 (1955). - 6. Harris, H. und E. B. Robson, Biochim. bio- physica Acta Amsterdam 73, 649 (1963). - 7. Harris, H., D. A Hopkinson und E. B. RoBson, Nature London 196, 1296 (1962). - 8. LaMotta, R. V., R.B. McComb, C. R. Noll jr., H. J. WeTstone und R. F. ReInfrank, Arch. Biochem. Biophysics 124, 299 (1968).

Dr. H. Weiß

X 1115 Berlin-Buch Lindenberger Weg 80 